\title{
Hélisenne de Crenne : traduire, réécrire, amplifier Virgile au XVI ${ }^{\mathrm{e}}$ siècle
}

\section{Ellen Delvallée}

\section{(2) OpenEdition}

12 Journals

Édition électronique

URL : http://journals.openedition.org/rhetorique/416

DOI : $10.4000 /$ rhetorique.416

ISSN : 2270-6909

Éditeur

UGA Éditions/Université Grenoble Alpes

Édition imprimée

ISBN : 978-284310-303-2

\section{Référence électronique}

Ellen Delvallée, « Hélisenne de Crenne : traduire, réécrire, amplifier Virgile au XVIe siècle », Exercices de rhétorique [En ligne], 5 | 2015, mis en ligne le 17 septembre 2015, consulté le 12 septembre 2020. URL http://journals.openedition.org/rhetorique/416 ; DOI : https://doi.org/10.4000/rhetorique.416

Ce document a été généré automatiquement le 12 septembre 2020.

\section{(c) (1) () (2)}

Les contenus de la revue Exercices de rhétorique sont mis à disposition selon les termes de la Licence Creative Commons Attribution - Pas d'Utilisation Commerciale - Partage dans les Mêmes Conditions 4.0 International. 


\title{
Hélisenne de Crenne : traduire, réécrire, amplifier Virgile au XVI siècle
}

\author{
Ellen Delvallée
}

1 Marguerite Briet (vers 1510-vers 1560), alias Hélisenne de Crenne, est une femme de lettres originaire de Picardie connue pour ses œuvres sentimentales et moralisantes, telles que Les angoysses douloureuses qui procedent d'amours (Paris, D. Janot, 1538, rééd. Paris, P. Sergent, 1541) ou Les Epistres familieres et invectives (Paris, D. Janot, 15391). Nul doute que sa traduction de l'Énéide (Paris, D. Janot, 1541) et en particulier du livre IV - que nous éditons - dans lequel est narré le séjour du héros troyen à la cour de Didon, est issue de cet intérêt pour les histoires d'amour pathétiques décrites du point de vue des femmes.

2 Le livre IV seul fut plusieurs fois traduit ou réécrit au XVI siècle : on peut rappeler la traduction de Du Bellay, assortie de l'épître d'Ovide de Didon à Énée ${ }^{2}$, la réécriture théâtrale de Jodelle, Didon se sacrifiant ${ }^{3}$, ou celle romanesque, de Denisot ${ }^{4}$. La traduction de Crenne se distingue en raison de son français constamment latinisé. On s'habitue du reste assez vite à cette langue particulière, surtout si on a un œil sur le texte latin de Virgile : ainsi la « cure » du tout début est le fameux cura qui termine le premier vers du livre IV, et la " pyre », la pyra ou bûcher du vers 504. En revanche, la traduction de Crenne partage avec les autres traductions et réécritures de son temps une certaine liberté par rapport au texte source, qu'elle développe en bien des endroits. Arrêtonsnous sur le sens de ces amplifications.

\section{Les amplifications}

3 Geste rhétorique défini ${ }^{5}$, l'amplification ne renvoie pas qu'aux ajouts d'Hélisenne de Crenne portés au texte de Virgile : sous cette appellation figurent toutes les techniques par lesquelles la traductrice souligne l'importance de certains aspects du texte de Virgile, le commente, l'oriente vers une lecture érudite ou féministe. L'amplification 
rhétorique ne s'appuie pas sur un critère de longueur quantitative mais d'importance qualitative. Aussi les amplifications d'Hélisenne de Crenne peuvent-elles parfois se trouver dans ce qu'elle ne traduit pas, ou qu'elle évoque plus brièvement, afin de souligner un autre fait. Dans sa traduction de l'Énéide, les amplifications portent essentiellement sur trois points: précisions documentaires, effets pathétiques, commentaires rhétoriques.

\section{Les précisions documentaires}

Hélisenne de Crenne, dans le corps de sa traduction mais surtout dans les manchettes ajoutées, s'efforce souvent de spécifier le personnel ou la géographie mythique que convoque Virgile dans son récit. Ainsi Fame (Fama, la Renommée) se trouve-t-elle précisément décrite au chapitre IX, en vertu d'une amplification reposant sur la définition du personnage par son origine. Les multiples manchettes portant sur les divinités les définissent, expliquent leurs noms et leurs attributs. Il en va de même des toponymes. Évoquons enfin les énumérations par lesquelles Hélisenne de Crenne spécifie les sous-catégories d'une entité donnée, telles que les différents dieux sylvestres (chapitre VIII) ou les différents noms de Phébus ou de la Lune (chapitre XXIX). Définition et spécification sont donc autant de procédés amplificatoires faciles à repérer par lesquels la traductrice souligne l'aspect antique du texte source et suggère une lecture promouvant un savoir humaniste. Les innombrables latinismes employés par Hélisenne de Crenne ont, d'une certaine façon, un effet semblable, et, quoique n'augmentant pas la longueur de la traduction, ils peuvent ainsi être considérés comme des amplifications lexicales.

\section{Les effets pathétiques}

5 Comme le précise Christiane Louette dans son étude sur la réécriture du livre IV de l' Énéide par Denisot ${ }^{6}$, les doublons synonymiques, hérités du Moyen Âge et caractéristiques de la prose du $\mathrm{XVI}^{\mathrm{e}}$ siècle, sont à la fois des ornements et des procédés amplificatoires servant souvent à accroître le pathos d'une description. Cette analyse est parfaitement applicable à Hélisenne de Crenne. Citons quelques exemples : "griève et précipiteuse cure" (chapitre premier), "affliction et amertume extrême" (chapitre XV), "plaintes et exclamations piteuses » (chapitre XXV); ces deux derniers exemples montrent par ailleurs que l'emploi de l'hyperbole vient souvent en renfort du pathos. Des ajouts relevant de l'hypotypose servent aussi à souligner le désordre physique des personnages, traduction de leurs conflits moraux ou de leurs passions. L'amplification porte alors moins sur les mots que sur les idées. Il en va ainsi, notamment, des cheveux d'Énée qui se dressent sur sa tête peu après la visite nocturne de Mercure (chapitre XIII), du spectacle des préparatifs du départ d'Énée qui redouble la tristesse de Didon (chapitre XIX) ou encore de la scène dans laquelle Anne tient dans ses bras sa sœur agonisante, salissant ses habits, impuissante face aux sursauts de Didon (chapitre XXXIII). Là encore nous laissons au lecteur sensibilisé sur ce point le soin de repérer toutes les occurrences de ce procédé. Grâce à l'amplificatio verborum autant que rerum, Hélisenne de Crenne met en valeur les ressources pathétiques du texte de Virgile. 


\section{Les commentaires rhétoriques}

6 Les termes introducteurs des discours, ou encore les verbes désignant l'acte de parole au sein d'un discours ne sont jamais anodins : ils définissent, rhétoriquement, le type de discours que prononce le personnage, clarifient ses intentions. Absents du texte latin, ils montrent, en français, que la traduction n'est pas possible sans une analyse rhétorique préalable, grâce à laquelle chaque discours est caractérisé par son but et par l'éthos des personnages qu'il met en scène. C'est ce que démontrent parfaitement les articles de Christiane Deloince-Louette sur Denisot ou de Francis Goyet sur Didon se sacrifiant ${ }^{7}$. Rappelons par ailleurs qu'au Moyen Âge, la traduction et le commentaire (rhétorique) sont un seul et même geste. Les pratiques ne se dissocient que dans le courant du $\mathrm{XVI}^{\mathrm{e}}$ siècle ${ }^{8}$ : aussi n'est-il guère surprenant de voir encore les traces de cette confusion dans les traductions de Virgile, un des auteurs antiques les plus cités en exemple dans les manuels de rhétorique. Ainsi, Hélisenne de Crenne traduit et analyse son texte en même temps. Par exemple, Anne "obsècre» et "suade" (texte du chapitre III) sa sœur de retenir les Troyens à Carthage : l'ajout de ces termes montre que sa réponse à l'éloge d'énée par Didon (qui l'« extolle » au chapitre II) n'appartient pas au genre démonstratif mais bien au délibératif, et relève du conseil voire de l'exhortation («instigue et exhorte » au titre même du chapitre III). De même, le titre du chapitre XV indique clairement que le premier discours de Didon à Énée est de l'ordre du reproche ("elle l'incrèpe »); la réponse de celui-ci, qui ne veut pas «être contaminé de vitupérable vice d'ingratitude ", se présente comme une "excuse », en vertu de laquelle la faute est reportée sur les dieux, qui lui ordonnent de partir (chapitre XVI) ; enfin celle-ci, réfutant la validité de ces excuses, s'abandonne aux reproches «véhémen(ts)» et aux «plaintes » envers ces mêmes dieux (chapitre XVII). « Reproches " (plus ou moins véhéments selon que le locuteur cherche encore, ou non, à maintenir l'alliance avec son interlocuteur) et « excuses » n'apparaissent pas dans le texte de Virgile et sont autant de types de discours codifiés, qu'Hélisenne de Crenne désigne ici explicitement ${ }^{9}$. Nous pourrions encore évoquer les nombreuses " plaintes ", « complaintes » ou « lamentations » d'Iarbas ou de Didon, qui renvoient également à un type de discours répertorié. L'amplification, lorsqu'elle porte sur des discours, est donc souvent la trace d'une analyse rhétorique du modèle virgilien.

7 Ces deux derniers aspects de l'amplification convergent vers une même fin : proposer, à l'instar de Jodelle et de Denisot, une lecture pro-Didon du livre IV, dans laquelle Énée ne peut plus guère invoquer la volonté des Dieux pour excuser son départ (argument minimisé au chapitre XVI) et où son ingratitude est manifeste face aux bienfaits que rappelle Didon (d'ailleurs amplifiés par l'énumération au chapitre XVII). L'auteur des Angoysses douloureuses qui procedent d'amours se montre naturellement sensible au sort qui accable Didon, aux doutes amoureux qui frappent Énée (chapitres XIII et XV), et moins, dans ce livre IV, au récit des aventures du héros fuyant Troie pour l'Italie. Hélisenne de Crenne fait de Didon un personnage pathétique non seulement victime d'Énée, mais peut-être aussi l'interlocutrice malheureuse d'une sœur qui l'a persuadée à retenir le héros troyen alors que la reine de Carthage était résolue à rester veuve.

Dans la traduction d'Hélisenne de Crenne, l'amplification n'est pas qu'un ornement: c'est un geste rhétorique qui fait signe vers une lecture documentaire, pathétique et volontiers féministe du livre IV de l'énéide. L'attention portée à ce procédé montre 
combien cette traduction s'apparente plutôt à une réécriture, indissociable des autres œuvres romanesques et épistolaires d'Hélisenne de Crenne.

\section{Principes d'édition} des Eneydes du treselegant poete Virgile, traduictz de latin en prose françoyse par ma dame Helisenne, Paris, D. Janot, 1541. Nous reproduisons ici sa traduction pour le seul livre IV, en établissant le texte à partir de l'exemplaire très lisible de la BnF (Arsenal, Rés. FOLBL-613). Les métacatalogues n'indiquent qu'un seul autre exemplaire, que nous n'avons pas vu, à la Bibliothèque de Genève (cote Hd 91). Nous n'avons pu reproduire les belles vignettes sur bois qui scandent la traduction. Nous remercions très vivement Michèle Clément et de nous avoir signalé cette traduction et de nous avoir fourni le tirage papier dont elle disposait, à partir d'un microfilm de la BnF (où quelques taches illisibles sont dues aux rousseurs de l'exemplaire de l'Arsenal). Notre texte respecte les lignes de l'original, afin d'en faciliter la comparaison.

Chapitrage : nous ajoutons entre crochets droits, après chaque indication de chapitre, la référence à l'Énéide (abrégée en Aen.). Nous remplaçons le chiffre romain IIII par IV, et corrigeons l'erreur de numérotation de chapitres intervenue entre les chapitres XV et XVIII (le chap. XVI étant numéroté « IX » et le XVII, « XV »). Nous mettons en gras les sommaires de chaque chapitre, pour les distinguer plus nettement du texte même. Enfin, nous donnons en minuscules la première ligne de chaque chapitre (que l'édition de 1541 donne en majuscules), puis ajoutons les guillemets pour distinguer les discours de personnages.

11 Nous corrigeons les erreurs manifestes, que voici dans leur ordre d'apparition (outre de nombreux que que nous remplaçons par qui) : «c'est » pour «cet» (et inversement), « es" pour «as ", «se " pour «ce», « entrenir » pour «entretenir», " compagnée » pour «compagnie », "procrée » pour " procréée ", «t'auront nous » pour «t'auronsnous », "plusillanimes» pour «pusillanimes», «vierge» pour «verge», « inquietitude » pour « inquiétude ", " mortifere » pour " mortifères », « juge-gement " (lors d'un saut de ligne) pour «jugement», «qu'elle» pour «quelle», «n'y " pour «ni », «specule» pour «specules», «donne» pour «donnes», «punitions» pour «punition », «qu'ilz» pour «qui », « d'eust » pour « dût », « casse » pour « cassée », « appelée » pour « appelé », «si » pour «s'y »; enfin nous rétablissons la parenthèse ouverte lorsqu'elle est manquante. Au-delà de ces erreurs manifestes, nous avons aussi corrigé "ses temples» en "tes temples» (f. $35 \mathrm{r}^{\circ}$ ), «lignée Cyllène » en "lignée de Cyllène » $\left(f .36 v^{\circ}\right)$, «Parquoi » en " Pourquoi » (f. $\left.97 v^{\circ}\right)$, « d'aucuns y est ajouté » en "d'aucuns y ont ajouté » (f. $102 \mathrm{r}^{\circ}$, en note). Enfin, l'original inverse de façon assez fréquente l'usage moderne du $s$ final pour les verbes à la $2^{\mathrm{e}}$ personne du singulier, en le mettant là où nous ne le mettons pas et vice-versa : nous suivons l'usage actuel.

Les graphies sont modernisées, mais la ponctuation et la casse sont respectées. Nous modernisons de même la graphie des noms propres de personnes, peuples ou divinités ainsi que les toponymes, en suivant la graphie et les formes de l'édition de l'Énéide aux C. U. F. (éd. et trad. Jacques Perret, Paris, Les Belles Lettres, 1995 [1977]) : au lieu de « Hiarbas », nous écrivons « Iarbas »; au lieu de « Xanthus », le "Xanthe », etc. Cela ôte parfois un peu de son charme au texte de Crenne, et nous invitons le lecteur à revenir de temps en temps au texte dans sa version originale, disponible sur le site de 
l'équipe ${ }^{10}$, pour y retrouver par exemple "la belle Aurora", plutôt que notre "belle Aurore ", ou «la nuict avec son tenebreux vmbre» plutôt que «la nuit avec sa ténébreuse ombre ».

Au-delà des noms propres, nous allons en effet jusqu'à de fortes modifications de grammaire et de morphologie, le but étant que le lecteur peu initié au XvI ${ }^{\mathrm{e}}$ siècle ne soit pas submergé par les formes latino-françaises de Crenne. Grammaire : nous modifions le genre des substantifs lorsque ceux-ci sont employés dans le genre qui n'est plus celui $\mathrm{du}$ français moderne, accordant affaire et ombre au féminin; navires, labeurs, ouvrage, espace, ordre, reproches, gestes, office, au masculin. Morphologie : affermée est remplacé par affirmée, amaritude par amertume, angustie (et ses dérivés) par angoisse (et ses dérivés), apparoir par apparaître, architecteurs par architectes, assiduellement par assidûment, benevolence par bienveillance, clairs voyans par clairvoyants, claritude par clarté, comparoir par comparaitre, crédence par croyance, crudélité par cruauté, eversera par renversera, extinte par éteinte, fatigieulx par fatigants, innumerable par innombrable, instinction par instinct, interpreteur par interprète, inuadez par envahis, maledite par maudite, malevolence par malveillance, obténébré (et ses dérivés) par enténébré (et ses dérivés), ouvré par ouvragé, pourmenant par promenant, poursuivir par poursuivre, pourtraictes par portraites, profondité par profondeur, inobediens par inobéissants, réginale par royale, reluscence par reluisance, repulser (et les formes conjuguées) par repousser, sçelere (et ses dérivés) par scélérat (et ses dérivés), seminer par semer, splendissant par resplendissant, syncopice par syncope, tarde par tardive.

En revanche, nous gardons les adjectifs et pronoms démonstratifs iceux, cestuy, et leurs composés (mais nous remplaçons l'adverbe cy par ici), devant l'impossibilité de les moderniser sans en altérer certaines valeurs sémantiques ou référentielles.

Enfin, pour les mots que nous ne modifions pas et qui appellent une note, nous nous limitons au strict minimum, et nous ne répétons pas la note à la réapparition du même mot, afin de ne pas alourdir la présentation. Ces notes relèvent d'un simple glossaire ; nous avons écarté des notes proprement littéraires.

\section{Table des chapitres}

- Chapitre I. [Aen. IV, 1-5]: Comme Didon écoutant cette accommodée narration, souffrait pour amour vénérique une peine intolérable, et quand l'heure tardive la stimula, en sa chambre ornée se retirer, ne fut en sa faculté de repos prendre, parce que trop était occupée à méditer et penser.

- Chapitre II. [Aen. IV, 6-30] : Didon persistant en l'amoureuse flamme devise avec sa sœur Anne, extollant merveilleusement la modestie et discrétion d'Énée, lequel elle déclare être celui seul qui a rendu sa chaste pensée vacillante: Toutefois le tout bien recogité, elle conclut sa viduité observer.

- Chapitre III. [Aen. IV, 31-53]: Anne répondant à Didon, allègue pluralité de propos : par lesquels elle l'instigue et exhorte qu'ainsi seule ne consume sa florissante jeunesse, concluant qu'elle se doit avec Énée par matrimoniale alliance associer.

- Chapitre IV. [Aen. IV, 54-89]: Comme Didon étant ainsi persuadée, de plus en plus s'enflamme, expulsant de son cœur amoureux toute honte, et comment elle se transmigra au temple en la société sororale, où elle fit sacrifice. Et des gestes et contenances qu'elle tenait, et aussi des visions nocturnes qu'amour lui représentait. 
- Chapitre V. [Aen. IV, 90-107] : Comme la déesse Junon ayant intelligence que Didon était de la fureur d'amour exagitée, prémédita d'y obvier, et adressa à Vénus son subtil parler, l'exorant que consentir se veuille que d'Énée et de Didon la nuptiale conjonction soit consommée.

- Chapitre VI. [Aen. IV, 107-114] : Vénus répondant à Junon, simule acquiescer à sa requête, disant que grande lætification lui sera, si Troyens et Tyriens vivent en perpétuelle concorde.

- Chapitre VII. [Aen. IV, 115-128] : Junon estimant les paroles de Vénus être avec vérité proférées, lui narre le subtil moyen, par lequel Énée et Didon se pourront en alliance conjugale confermer.

- Chapitre VIII. [Aen. IV, 129-172] : Comme Énée et Didon allèrent aux champs pour prendre soulacieux exercice : mais la survenue de la diversité du temps tant les atédia, que pour obvier à la merveilleuse inondation, dedans spelunque occulte s'y réduirent, qui fut occasion de grande infélicité.

- Chapitre IX. [Aen. IV, 173-205] : De la vélocité et promptitude de Fame mauvaise, laquelle selon les antiques fut de la déesse Cybèle procréée, et lui propina grande agilité pour plus facilement en diversités de lieux toutes nouvelles annoncer : Par quoi icelle Fame ne voulant occulter la faute par Didon perpétrée, en tous lieux de Lybie la fit savoir : Et mêmement au Roi Iarbas qui en fut fort irrité, et en fit à Jupiter piteuse complainte.

- Chapitre X. [Aen. IV, 206-218] : La piteuse complainte faite, par le Roi Iarbas à l'altitonnant Jupiter.

- Chapitre XI. [Aen. IV, 219-237] : Comme Jupiter ayant intelligence de la juste indignation du Roi Iarbas y voulut pourvoir de remède, donnant charge expresse au très éloquent Mercure de se transmigrer vers Énée, pour lui dire et remontrer que l'urgente nécessité requiert que promptement de Carthage s'absente.

- Chapitre XII. [Aen. IV, 238-278] : Comme Mercure obéissant au commandement de Jupiter, se transmit dedans Carthage où il référa à Énée les paroles de l'altitonnant Jupiter : puis incontinent ledit Mercure par l'air spacieux s'évanouit.

- Chapitre XIII. [Aen. IV, 279-295]: Comme Énée pour les paroles ouïes fut en grande perplexité, et lui survinrent variétés de pensées, pour le débat que raison et amour lui inféraient : toutefois après diverses disputations, convoqua aucuns des siens plus fidèles, et conclut occultement se départir.

- Chapitre XIV. [Aen. IV, 296-304] : Comme la Reine de la future absence s'aperçut : dont elle fut de si précipiteuse ire commue, qu'elle en perdit toute modestie et contenance.

- Chapitre XV. [Aen. IV, 305-333] : Des propos que tint l'anxieuse Didon à Énée, par lesquels elle l'incrèpe du vice d'infidélité, et avec diversités de paroles plusieurs choses lui remontre.

- Chapitre XVI. [Aen. IV, 333-364] : La réponse faite par Énée à Didon, par laquelle il l'assure qu'il aura assidue et continuelle recordation des biens et gratuités qu'il a d'elle en grande abondance reçus, Toutefois il lui nie qu'alliance nuptiale entre eux soit faite : Et d'avantage dit que pour plusieurs occasions légitimes est stimulé la région Italique investiguer.

- Chapitre XVII. [Aen. IV, 365-391] : Didon voyant la détermination irrévocable de son amant, donna principe à plusieurs rigoureux propos, qui furent avec grande véhémence prononcés : Et après y avoir imposé fin, par superabondante angoisse tomba pâmée.

- Chapitre XVIII. [Aen. IV, 391-407] : Comme Didon étant en cette syncope, fut conduite en sa chambre. Et lors Énée considérant les anxiétés d'icelle, s'efforce avec son artificielle éloquence d'aucunement les angoisses d'elle mitiguer : puis après diversité de pensées, il commença de donner ordre à son absence. 
- Chapitre XIX. [Aen. IV, 408-415] : De la peine mentale qui pouvait agiter le délicieux cœur de Didon, en contemplant les préparatifs d'absence. Et comment amour, qui en elle dominait et seigneuriait, l'instigua d'expérimenter d'avoir recours aux humbles et instantes prières.

- Chapitre XX. [Aen. IV, 416-436] : Comme Didon parlant à sa sœur Anne, doucement l'exore que transmigrer se veuille vers Énée, pour lui remontrer avec paroles suaves et melliflues, que pour quelque temps veuille sa future absence différer.

- Chapitre XXI. [Aen. IV, 437-449]: De la diligence que fit Anne pour la volonté sororale accomplir : Mais Énée résistant à toutes persuasions, ne lui voulut aucune audience donner.

- Chapitre XXII. [Aen. IV, 450-477]: De l'extrême angoisse que souffrit Didon pour être d'espérance destituée, et de ce qu'il intervint au sacrifice qu'elle fit avec narration de ses anxieuses imaginations et furieuses pensées. Et de la détermination par elle prise pour ses jours anticiper.

- Chapitre XXIII. [Aen. IV, 478-498] : Comme Didon dissimulant sa délibération mortifère, persuade à sa sœur que l'user d'art magique sera apte pour le faux amant oublier : par quoi à sadite sœur obsècre préparer plusieurs choses pour sacrifier, qu'elle dit être ordonnées par celle de qui l'art d'elle veut ensuivre.

- Chapitre XXIV. [Aen. IV, 499-503]: Anne ignorant la détermination de Didon, prépara promptement toutes choses, selon l'instruction donnée.

- Chapitre XXV. [Aen. IV, 504-533] : Des préparatifs que fit Didon simulant vouloir exhiber sacrifices. Et comment avec voix lamentable, elle invoquait les déifiques puissances.

- Chapitre XXVI. [Aen. IV, 534-552] : Didon en soi grièvement angoissée et adolorée fait ses piteuses complaintes avec diversité d'opinions : mais finalement se détermine à son propos mortel poursuivre.

- Chapitre XXVII. [Aen. IV, 553-570]: Comme l'annonciateur des dieux Mercure derechef s'apparut à Énée, l'admonestant que sans dilation se départe, et l'avertit que si promptement ne le fait, il pourra en grand péril succomber.

- Chapitre XXVIII. [Aen. IV, 571-583] : Comme après que se fut séparé Mercure, Énée excité du sommeil promptement se lève : et après avoir instigué ses gens leur référant les paroles annoncées, sans plus différer se départirent.

- Chapitre XXIX. [Aen. IV, 584-621] : Description du deuil extrême que fit l'angoissée Didon voyant l'absence de son ami Énée, et des gestes extérieurs d'elle. Et aussi des diversités de propos qu'amour fervente lui faisait furieusement prononcer.

- Chapitre XXX. [Aen. IV, 622-629] : Supplication de la part de Didon aux Tyriens.

- Chapitre XXXI. [Aen. IV, 630-641] : Didon ne pouvant plus tolérer les acerbes douleurs qui l'exagitent, prémédite le moyen de sa mort prochaine, pour auquel plus facilement parvenir, trouva manière de se séquestrer de toute société humaine.

- Chapitre XXXII. [Aen. IV, 642-662]: Comme Didon se trouvant de toute société humaine séparée, se réduit au lieu de son palais le plus taciturne: et des piteux regrets et exclamations qu'elle fit, se complaignant de la variété de Fortune, qui en cette extrémité l'avait constituée. Et comment imposant fin à son propos se donna le coup mortifère.

- Chapitre XXXIII. [Aen. IV, 663-692] : Des lamentations, lachrymes et pleurs, qui se firent pour l'infélicité de Didon : laquelle chose étant parvenue à la notice de sa sœur, fut plus que l'on ne pourrait exprimer, angoissée, et fit plusieurs regrets sur le corps d'icelle Didon: de laquelle la dolente âme n'était encore transmigrée.

- Chapitre XXXIV. [Aen. IV, 693-705] : Comme la sublime déesse Junon eut compassion de la trop longue peine de l'infélice dame: dont pour y imposer fin envoya du ciel Iris, lui donnant charge que de la prison corporelle délie l'âme. 


\section{NOTES}

1. Nous renvoyons aux introductions des éditions modernes de ces deux ouvrages (Les angoysses douloureuses qui procedent d'amours, éd.Ch. de Buzon, Paris, Champion, 1997 et Les Epistres familieres et invectives, éd. J. C. Nash, Paris, Champion, 1996) pour une plus ample description de la biographie et des œuvres d'Hélisenne de Crenne.

2. Joachim Du Bellay, Le quatriesme livre de l'Énéide traduict en vers françoys. La complaincte de Didon à Énée, prinse d'Ovide. Autres oeuvres de l'invention du translateur, Paris, Vincent Certenas, 1552.

3. Probablement écrite vers 1555, mais éditée de façon posthume avec les autres écrits de Jodelle dans Les Euvres et Meslanges Poëtiques d'Estienne Jodelle sieur du Lymodin, Paris, Nicolas Chesneau, 1574.

4. T. Valentinian [Nicolas Denisot], Histoire de l'Amant resuscité de la mort d'amour, Lyon, Maurice Roy et Louis Pesnot, 1558 ; éd. V. Duché-Gavet, Genève, Droz, 1998.

5. Sur ce sujet, voir S. Macé, "L'amplification, ou l'âme de la rhétorique. Présentation générale », Exercices de rhétorique [En ligne], 4 | 2014, mis en ligne le 12 décembre 2014, consulté le 11 mai 2015. URL : http://rhetorique.revues.org/364 ; DOI : 10.4000/rhetorique.364

6. C. Deloince-Louette, "De Virgile à Denisot : une pratique romanesque du commentaire rhétorique ", Rhétorique, poétique et stylistique (Moyen Âge-Renaissance), éd. A. Bouscharain et D. James-Raoul, Bordeaux, Presses Universitaires de Bordeaux (Eidôlon, $\mathrm{n}^{\circ}$ 112), 2014, p. 95-106.

7. Fr. Goyet, «La rhétorique de Didon dans l'acte II : l'exprobratio au sein des vers 851-952 ", Lectures d'Etienne Jodelle. Didon se sacrifiant, éd. E. Buron et O. Halévy, Rennes, P.U.R., 2013, p. 65-96. 8. Sur l'histoire de la traduction et le tournant de la Renaissance, voir notamment A. Berman, «De la translation à la traduction », TTR : traduction, terminologie, rédaction, vol. $1, \mathrm{n}^{\circ} 1,1988$, p. 23-40.

9. Pour un commentaire rhétorique de ces discours chez Virgile, voir C. Loutsch, «Énée face à Didon (Énéide IV, 333-361) », Exercices de rhétorique [En ligne], 2 |2013, mis en ligne le 17 octobre 2013, consulté le 12 mai 2015. URL : http://rhetorique.revues.org/179; DOI : 10.4000/ rhetorique.179. Voir également, sur la réécriture de ce passage central du livre IV, les articles de C. Deloince-Louette et de Fr. Goyet mentionnés ci-dessus.

10. Voir la page dédiée à l'édition sur le site de l'équipe RARE Rhétorique de l'Antiquité à la Révolution, URL : http://rare.u-grenoble3.fr/spip/spip.php?article415.

\section{AUTEUR}

\section{ELLEN DELVALLÉE}

Univ. Grenoble-Alpes - LITT\&ARTS / RARE Rhétorique de l'Antiquité à la Révolution 